\title{
Developmental course of subclinical positive and negative psychotic symptoms and their associations with genetic risk status and impairment
}

\author{
Mayke Janssens ${ }^{\mathrm{a}, \mathrm{d}, 5}$, Lindy-Lou Boyette ${ }^{\mathrm{b}, \mathrm{e}, 5}$, Henriëtte D. Heering ${ }^{\mathrm{b}}$, Agna A. Bartels-Velthuis ${ }^{\mathrm{c}}$, Tineke Lataster ${ }^{\mathrm{a}}$, \\ Genetic Risk and Outcome of Psychosis investigators
}

\section{René S. Kahn ${ }^{3}$, Lieuwe de Haan ${ }^{2}$, Jim van Os ${ }^{1}$, Durk Wiersma ${ }^{4}$, Richard Bruggeman ${ }^{4}$, Wiepke Cahn ${ }^{3}$, Carin Meijer ${ }^{2}$, Inez Myin-Germeys ${ }^{1,6}$}

${ }^{1}$ Maastricht University Medical Center, South Limburg Mental Health Research and Teaching Network, Maastricht, The Netherlands

${ }^{2}$ Academic Medical Center University of Amsterdam, Department of Psychiatry, Amsterdam, The Netherlands

${ }^{3}$ Department of Psychiatry, Rudolf Magnus Institute of Neuroscience, University Medical Center Utrecht, Utrecht, The Netherlands

${ }^{4}$ University of Groningen, University Medical Center Groningen, University Center for Psychiatry, Groningen, The Netherlands

a Maastricht University Medical Center, South Limburg Mental Health Research and Teaching Network, Maastricht, The Netherlands

${ }^{\mathrm{b}}$ Academic Medical Center University of Amsterdam, Department of Psychiatry, Amsterdam, The Netherlands

c University of Groningen, University Medical Center Groningen, University Center for Psychiatry, Groningen, The Netherlands

${ }^{\mathrm{d}}$ Faculty of Psychology and Educational Sciences, Open University of the Netherlands, Heerlen, the Netherlands

e Faculty of Clinical Psychology, University of Amsterdam, Amsterdam, The Netherlands

\section{A R T I C L E I N F O}

\section{Article history:}

Received 4 September 2015

Received in revised form 21 March 2016

Accepted 24 March 2016

Available online $\mathrm{xxxx}$

\section{Keywords:}

Psychotic experiences

Schizotypy

Attenuated symptoms

Schizophrenia relatives

Proneness-persistence-impairment model

Continuum model

\begin{abstract}
A B S T R A C T
The proneness-persistence-impairment (PPI) model states that psychotic experiences are more likely to lead to impairment if their expression becomes persistent. Higher genetic risk for psychosis is known to affect proneness and persistence of subclinical positive symptoms. Less is known about potential effects of genetic risk on the course of subclinical negative symptoms, impairment, and their subsequent associations. The current study examined these issues in a large sample $(n=1131)$, consisting of individuals with higher genetic risk (siblings of patients with psychotic disorders, $n=703$ ) and lower genetic risk (controls without a family member with lifetime psychosis, $n=428$ ). Psychotic experiences were assessed with the CAPE questionnaire, at two time points three years apart. Participants were allocated to one of four groups representing developmental course: stable low, decreasing, increasing or persisting subclinical positive/negative symptoms. Lifetime clinical psychosis was an exclusion criterion at baseline. Higher genetic risk status was found to be associated with a persisting course of both subclinical positive and negative symptoms, symptom-related distress and functional impairment. There is no evidence for an effect of genetic risk status on the association between developmental course and impairment. The results of the current study underline the importance of assessing psychotic experiences in the context of genetic risk, multidimensional and over time. Additionally, the current findings both underscore and contribute to the PPI model: psychotic experiences are more likely to lead to impairment if their expression becomes persistent, both in individuals with higher and lower genetic risk for psychosis.
\end{abstract}

(c) 2016 Published by Elsevier B.V.

\section{Introduction}

Subclinical manifestations of the psychosis phenotype, also referred to as psychotic experiences, are found to be present in the general population with prevalence rates ranging up to $17.5 \%$ (Van Os et al., 2009). Studies often use the term 'psychotic experiences' interchangeably with a multitude of other terms all developed to

\footnotetext{
${ }^{5}$ Joined first authors.

${ }^{6}$ Corresponding author.
}

reflect vulnerability for schizophrenia-spectrum pathology, such as 'psychotic-like experiences', 'subclinical psychotic symptoms', 'schizotypy', 'schizotypical symptoms', 'psychosis-proneness' and 'attenuated psychotic symptoms' (Kwapil and Barrantes-Vidal, 2015). In the current study we choose to use the term psychotic experiences, defined as multidimensional (including both subclinical positive and negative psychotic symptoms) psychometric risk for psychosis based on the Community Assessment of Psychic Experience (CAPE; www.cape42.homestead.com), a reliable and valid self-report questionnaire for dimensions of psychotic experiences in non-clinical samples (Konings et al., 2006). 
Findings of large general population studies show that for the vast majority of people, estimated between $75 \%$ and $90 \%$ (Van Os et al. 2009), psychotic experiences are transient and not indicative of need for care (Hanssen et al., 2005; Linscott and van Os, 2013). A minority of individuals, on the other hand, will make a transition to clinical psychosis, with an estimated prevalence rate around 4\% (Van Os et al., 2009). The proneness-persistence-impairment model of psychotic disorders states that the transition from psychotic experiences to clinical psychosis is preceded by the persistence of psychotic experiences and is accompanied by increasing impairment, depending on additional risk factors (Cougnard et al., 2007; Van Os et al., 2009). Previous research has indeed demonstrated that persistence of psychotic experiences is associated with transition to clinical psychosis and/or need for care (Cougnard et al., 2007; Dominguez et al., 2011; Wigman et al., 2011a; Wigman et al., 2011b) as well as functional impairment (Dominguez et al., 2011; Kaymaz et al., 2012; De Loore et al., 2011; Rossler et al., 2007; Wigman et al., 2011b). Moreover, in a cohort of adolescents progressively stronger associations with severity of psychotic experiences, symptom-related distress and functional impairment have been reported for respectively a stable low, decreasing, increasing and persisting trajectory of psychotic experiences (Wigman et al., 2011b).

However, studies focusing on the temporal course of psychotic experiences in non-clinical samples have almost exclusively focused on the subclinical manifestation of the positive symptom dimension of psychotic disorders: delusional ideation and perceptual anomalies. There is indication that the negative symptom dimension of psychotic disorders, consisting of symptoms such as blunted affect and avolition, is also present in the general population in attenuated forms (Maric et al., 2004, Stefanis et al., 2002). Furthermore, there is evidence for familial liability between subclinical and clinical expressions of both the positive and the negative symptom dimension of psychosis (Fanous et al., 2001; Lataster et al., 2014).

Subclinical negative psychotic symptoms (from here-on referred to as 'negative symptoms') are clinically relevant, as they are predictive of onset of clinical psychosis (Demjaha et al., 2012; Valmaggia et al., 2013), mental health care use (Dominguez et al., 2011; Maric et al., 2004) and functional impairment (Cohen and Davis, 2009; Dominguez et al., 2011; Kwapil et al., 2013), independent of subclinical positive psychotic symptoms (from here-on referred to as 'positive symptoms'). Additionally, there is indication of added value in assessing the course of negative symptoms over time, as the persistence of negative symptoms has been found to predict the onset and persistence of positive symptoms, and particularly their co-occurrence is found to predict helpseeking behaviour and dysfunction due to symptoms (Dominguez et al., 2010).

Previous research has shown that exposure to environmental risk factors such as childhood trauma (Read et al., 2005), cannabis use (Moore et al., 2007) and an urban environment (Krabbendam and van Os, 2005), increase the risk of persistence of positive symptoms in the general population (Cougnard et al., 2007). With regard to genetic risk, prior research in a general population female twin-sample has indicated genetic factors in the early prevalence of positive symptoms (Lataster et al., 2009a), and in particular a substantial genetic contribution to the persistence of positive symptoms is found (Wigman et al., 2011a). Dominguez et al. (2010) found indication for differences in risk factors between positive and negative symptoms in a non-clinical sample of adolescents and young adults: positive symptoms were found to be associated with environmental exposures, such as trauma and cannabis use, whereas negative symptoms were found to be associated with a pattern of socio-demographic associations possibly indicative of earlier developmental impairment, such as younger age and low education. These findings lead Dominguez et al. (2010) to hypothesize that genetic vulnerability and abnormal brain development may particularly drive the persistence of negative symptoms. In studies using variants of the schizotypy construct, the contribution of genetic factors to both the positive and negative symptom dimension is a well-established fact (e.g. Kendler et al., 1995).

In summary, findings of previous studies in non-clinical samples provide evidence that genetic risk for psychosis affects the proneness to and persistence of positive symptoms. To a lesser degree, there is indication for a contribution of genetic risk to the developmental course of negative symptoms. In addition, proneness to and persistence of both positive and negative symptoms are suggested to independently predict clinical and functional impairment. No studies to date have focused on the contribution of genetic risk to the relation between course of psychotic experiences and clinical and functional impairment.

The current study aims to replicate findings of previous studies regarding proneness and persistence of psychotic experiences, and aims to expand on previous studies by investigating a plausible genetic contribution to the proneness-persistence-impairment model, separate for positive and negative symptoms. To fulfil these aims, we examined associations between genetic risk status, the (three year) course of positive and negative symptoms and clinical and functional impairment in a large non-clinical sample, consisting of siblings of patients diagnosed with a psychotic disorder (representing higher genetic risk status for psychosis) and healthy controls without a first degree family member with a psychotic disorder (representing lower genetic risk status for psychosis). Clinical impairment is defined as symptom-related distress and transition to clinical psychosis. Functional impairment is defined in terms of social functioning and subjective quality of life. It was expected that (1) individuals with a higher genetic risk for psychosis (siblings) are more likely to experience a persistent course of both positive and negative symptoms compared to individuals with lower genetic risk (controls), (2) higher genetic risk status for psychosis (sibling status) predicts higher levels of clinical and functional impairment, (3) persistent course of both positive and negative symptoms independently predict higher levels of clinical and functional impairment, (4) the association between persistent course and impairment is more pronounced in individuals with a higher genetic risk status (siblings) compared to individuals with a lower genetic risk (controls).

\section{Methods}

\subsection{Participants and procedures}

Data pertain to the baseline and follow-up measure of the GROUP (Genetic Risk and Outcome of Psychosis) study, an ongoing longitudinal multicenter cohort study (for details see Korver et al., 2012). For the current study, only sibling- and healthy control-data were used. Participants were recruited in selected geographical areas in the Netherlands and Belgium. Inclusion criteria were: (i) age between 16 and 50 years, (ii) good command of the Dutch language, (iii) being able and willing to give informed consent. Siblings had a brother or sister meeting the DSM-IV-TR (APA, 2000) criteria for a non-affective psychotic disorder. Siblings were not allowed to meet criteria for a lifetime diagnosis of any psychotic disorder at baseline. Healthy control participants had no lifetime diagnosis of a psychotic disorder at baseline and no firstdegree relative with a lifetime psychotic disorder. The standing ethics committee approved the study, and all the subjects gave written informed consent in accordance with the committee's guidelines.

\subsection{Measures}

\subsubsection{Developmental course of psychotic experiences}

Psychotic experiences (from hereon: PE) were assessed with the self-report questionnaire Community Assessment of Psychic Experience (CAPE; www.cape42.homestead.com). Studies using the CAPE in general population samples have shown good psychometric properties in terms of reliability and validity (Konings et al., 2006). The CAPE positive and negative symptom frequency scales were used for analyses. The developmental course of PE was based on the presence or absence of high 
PE at baseline (lifetime presence) and (3-year) follow-up (interval presence), reflected in a mean score in the fourth quartile on the CAPE frequency scales, assessed for positive and negative symptoms separately. Cut-offs for positive and negative symptoms were resp. 0.31 and 0.72 at baseline, and 0.15 and 0.65 at follow-up. Participants were allocated to one of four groups representing developmental course: (0) stable low: high PE absent at both time points, (1) decreasing: high PE present at baseline but absent at follow-up, (2) increasing: high PE absent at baseline but present at follow-up, and (3) persistent: high PE present at both time points.

\subsubsection{Clinical outcome}

Transition to clinical psychosis was defined as the onset of florid psychotic symptoms (hallucinations, delusions, formal thought disorder and/or inappropriate/bizarre behavior), between baseline and followup, fulfilling DSM-IV criteria (APA, 2000) for a psychotic disorder as assessed by the Comprehensive Assessment of Symptoms and History (CASH; (Andreasen et al., 1992) at follow-up. For more details on criteria for transition to clinical psychosis we refer to Van Nierop et al. (2013). Overall symptom-related distress was assessed with the CAPE, based on the sum of positive, negative and depressive symptom distress scales, with higher scores reflecting more distress (range per item: 0-3, 20 items for the positive subscale, 14 items for the negative subscale and 8 items for the depressive subscale).

\subsubsection{Functional outcome}

Social functioning was assessed using the Social Functioning Scale (SFS) (Birchwood et al., 1990), a widely used instrument to measure areas of functioning essential for successful community maintenance. The total score on the SFS was used as a measure of overall social functioning in the past three months. Higher scores on the SFS indicate higher levels of social functioning (range: 93.36-134.93). The World Health Organization Quality of Life (WHOQOL-BREF; (WHO, 1998) was used to rate subjective quality of life (QoL) over the past two weeks. The sum score of the four domains (physical, psychological, social and environmental) was used, with higher scores reflecting better QoL (range: 26-130).

\subsection{Analyses}

Analyses were run using Stata, version 12 (Statacorp, 2011). We first investigated the association between genetic risk status and the developmental course of positive/negative subclinical symptoms. Multinomial logistic regression analyses were run using the MLOGIT command. Genetic risk status (lower risk (healthy controls): 0 vs. higher risk (siblings): 1) was used as independent variable and the developmental course of positive/negative symptoms as dependent variable. In Model 1 , age and gender were added as possible confounders. Also, the course of negative symptoms was added as covariate in the model of positive symptoms and vice versa (Model 2). As observations of individuals within the same family are more similar and residuals are not independent, cluster-robust standard errors were computed, using the CLUSTER option.

We then investigated the associations between genetic risk status and functional and clinical outcome. Linear regression analyses using the XTMIXED command were run, using genetic risk status as independent variable and functional (QoL or social functioning) or clinical outcome (overall symptom-related distress) as dependent variables, adding age and gender as covariates. Standard errors were corrected for hierarchical clustering of the data at the family-level.

In order to study the association between the developmental course of subclinical positive/negative symptoms on the one hand and functional or clinical outcome on the other, linear regression analyses with developmental course as independent variable and functional (QoL or social functioning) or clinical outcome (overall symptom-related distress) as dependent variable were run for the entire sample using the
XTMIXED command. Because there were relatively few individuals that transitioned to clinical psychosis, instead of allocating them to four groups, resulting in small or empty groups, we divided them into two groups (dichotomising the course-variable) resulting in a group with persisting symptoms and a group without persisting symptoms. For the analyses with transition as dependent variable, we performed logistic regression analyses with developmental course containing two levels (with/without persisting symptoms) as independent variable. Standard errors were corrected for hierarchical clustering of the data at the family-level. Results for the course of subclinical positive symptoms were corrected for the course of negative symptoms and vice versa. Also, age and gender were added as possible confounders. For the model of CAPE total distress (at follow-up), CAPE total distress at baseline was added as a covariate.

Finally, in order to assess the role of genetic risk status in the subsequent road to impairment, the group $\mathrm{x}$ developmental course interaction was tested in the different outcome models of positive symptoms and negative symptoms using linear regression analyses with the XTMIXED command as described above.

\section{Results}

\subsection{Sample}

Of the 1646 siblings and controls in the GROUP sample at baseline, baseline and follow-up CAPE scores were available for 1131 subjects ( 703 relatives and 428 controls). Due to missing values in the dependent variables or covariates, sample sizes for the analyses ranged from $\mathrm{n}=1007$ to $\mathrm{n}=1131$. See Table 1 for detailed sample characteristics. Mean time to follow-up was 2.9 years $(\mathrm{SD}=0.2$ ). A contingency table of the individuals in the positive and negative symptom categories is presented in Table 2a (for controls) and Table 2b (siblings).

\subsection{Effect of genetic risk on the course of psychotic experiences}

Significant associations were found between genetic risk status and the developmental course of PE when correcting for age and gender, with siblings experiencing higher levels of persisting courses of both positive and negative symptoms compared to controls (Table 3, Model 1 ). When additionally correcting for the alternate symptom cluster, only the association between genetic risk status and course of negative symptoms remained significant (Table 3, Model 2).

\subsection{The effect of genetic risk status on clinical and functional outcome}

Correcting for age and gender, significant associations were present between genetic risk status and functional as well as clinical outcome at follow-up. Genetic risk status was found to be associated with lower levels of social functioning $(\mathrm{B}=-1.78, \mathrm{P}<0.001,95 \% \mathrm{CI}=-2.58$; $-0.98)$, lower quality of life $(\mathrm{B}=-0.81, \mathrm{P}=0.04,95 \% \mathrm{CI}=-1.61$; $-0.01)$ and higher overall symptom-related distress $(\mathrm{B}=0.08, \mathrm{P}=$ $0.015,95 \% \mathrm{CI}=0.02 ; 0.15$ ) at follow-up. Siblings also showed a trend

Table 1

Sample characteristics (at baseline).

\begin{tabular}{lll}
\hline & Controls & Siblings \\
\cline { 2 - 2 } & $(\mathrm{n}=428)$ & \\
\hline Age (mean)(SD) & $31.3(10.7)$ & $27.8(8.0)$ \\
Gender $(\%$ male) & 56 & 55 \\
Education (\%) & & \\
$\quad$ No education & 0 & 0 \\
Primary school & 2 & 5 \\
Secondary school & 13 & 19 \\
High school & 31 & 21 \\
Vocational education & 44 & 42 \\
University & 10 & 13 \\
\hline
\end{tabular}


Table 2a

Number of individuals per developmental course for controls.

\begin{tabular}{llllllr}
\hline \multicolumn{7}{c}{ Negative symptoms } \\
\hline $\begin{array}{c}\text { Positive } \\
\text { symptoms }\end{array}$ & & Stable & Decreasing & Increasing & Persisting & Total N (\%) \\
& low & & & & \\
& Stable low & 350 & 22 & 22 & 2 & $\mathbf{3 8 5}(\mathbf{9 0 . 2})$ \\
& Decreasing & 15 & 1 & 1 & 2 & $\mathbf{1 9}(\mathbf{4 . 4})$ \\
& Increasing & 10 & 0 & 3 & 3 & $\mathbf{1 6}(\mathbf{3 . 7})$ \\
& Persisting & 4 & 1 & 1 & 1 & $\mathbf{7 ( 1 . 6 )}$ \\
& Total N & $\mathbf{3 7 9}$ & $\mathbf{2 4}(\mathbf{5 . 6})$ & $\mathbf{1 6}(\mathbf{3 . 7})$ & $\mathbf{8}(\mathbf{1 . 9})$ & $\mathbf{4 2 7}(\mathbf{1 0 0})$ \\
& $(\mathbf{\%})$ & $\mathbf{( 8 8 . 8 )}$ & & & & \\
\hline
\end{tabular}

towards an elevated risk of developing a clinical psychosis, though this effect did not reach statistical significance in the current sample ( $\mathrm{OR}=$ $2.2,95 \% \mathrm{CI}=0.47 ; 10.58$ ).

\subsection{Developmental course of psychotic experiences and clinical outcome}

When correcting for age, gender and the course of the alternate symptom cluster (negative symptoms), increasing subclinical positive symptoms were found to be associated with higher levels of overall symptom-related distress, and persisting subclinical positive symptoms were associated with transition to clinical psychosis (Table 4). For negative symptoms, increasing and persisting symptoms were associated with higher levels of overall symptom-related distress, independent of the course of positive symptoms. Persisting negative symptom course was not independently associated with transition to psychosis (Table 4).

\subsection{Developmental course of psychotic experiences and functional outcome}

Compared to the reference category (stable low) and corrected for age, gender and negative symptoms, we found increasing and persisting positive symptoms to be associated with both poorer social functioning and QoL at follow-up (Table 5). For negative symptoms, we found decreasing, increasing and persisting negative symptoms to be associated with poorer social functioning and QoL, independent of positive symptoms (Table 5).

3.6. The effect of genetic risk on the associations between course and outcome

When examining the interaction between genetic risk status and the developmental course of PE in the models of functional and clinical outcome, no significant interactions were found (results not shown). Controls and siblings did not differ significantly in the association between the course of positive or negative symptoms and any clinical or functional outcome measures. For the model of transition to clinical psychosis, the interaction could not be tested due to the small amount of individuals that transitioned ( $\mathrm{n}=10 ; 8$ siblings, 2 controls).

Table 2b

Number of individuals per developmental course for siblings.

\begin{tabular}{|c|c|c|c|c|c|c|}
\hline \multirow[b]{2}{*}{$\begin{array}{l}\text { Positive } \\
\text { symptoms }\end{array}$} & & \multicolumn{5}{|c|}{ Negative symptoms } \\
\hline & & $\begin{array}{l}\text { Stable } \\
\text { low }\end{array}$ & Decreasing & Increasing & Persisting & Total N (\%) \\
\hline & Stable low & 529 & 25 & 30 & 16 & $600(85.3)$ \\
\hline & Decreasing & 24 & 11 & 5 & 6 & $46(6.5)$ \\
\hline & Increasing & 11 & 3 & 7 & 6 & 27 (3.8) \\
\hline & Persisting & 9 & 5 & 3 & 13 & $30(4.3)$ \\
\hline & $\begin{array}{l}\text { Total N } \\
(\%)\end{array}$ & $\begin{array}{l}573 \\
(81.5)\end{array}$ & $44(6.3)$ & $45(6.4)$ & $41(5.8)$ & $703(100)$ \\
\hline
\end{tabular}

Table 3

Associations between genetic risk status and developmental course.

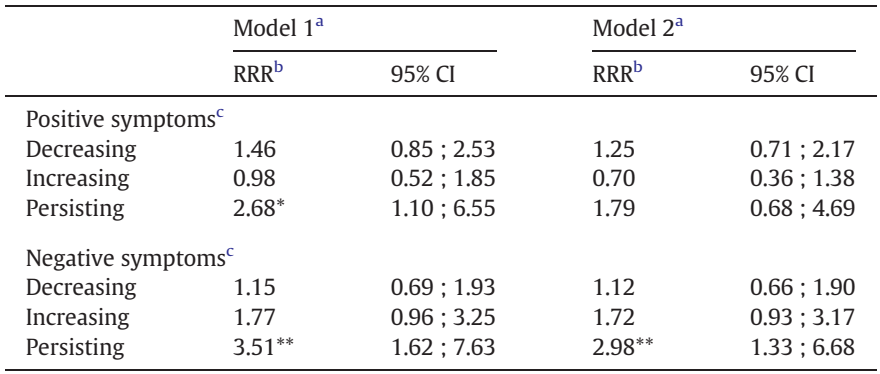

${ }^{*} \mathrm{P}<0.05,{ }^{* *} \mathrm{P}<0.01$

a Model 1: The association between genetic risk and course corrected for age, sex and for clustering at the family-level. Model 2: also corrected for the alternate symptom cluster.

b Relative Risk Ratio, representing the relative risk of experiencing a specific symptom course for individuals with higher genetic risk (siblings) relative to individuals with lower genetic risk (controls).

c Stable-low is used as the reference category

\section{Discussion}

The main aim of the present study was to investigate a plausible genetic contribution to the proneness-persistence-impairment model in a large non-clinical sample consisting of individuals with higher genetic risk (siblings) and lower genetic risk for psychosis (controls), separate for subclinical positive and negative symptoms. In line with our initial expectation, we found that siblings are more likely to experience a persistent course of both positive and negative symptoms compared to controls. Also, mostly in line with our initial expectations, we found that sibling status and persistent course of both positive symptoms and negative symptoms are associated with most types of clinical and functional impairment. However, the association between persistent course and impairment was not significantly different in siblings compared to controls.

\subsection{Genetic risk status predicts course of psychotic experiences}

In line with our first expectation, the findings of the current study show that both subclinical positive and negative symptoms are more likely to persist in siblings of patients with a psychotic disorder compared to controls. After correction for the alternate symptom cluster (in addition to age and gender), this association remained present for negative symptoms, but was no longer statistically significant for positive symptoms. This may suggest that genetic vulnerability particularly drives the course of negative symptoms, as has been proposed by Dominguez et al. (2010). A second explanation is that genetic risk may be (partly) associated with the co-occurrence of positive and negative symptoms, which is in line with a proposed multidimensional construct of subclinical expression of the psychosis phenotype (e.g. Kwapil

Table 4

Associations between developmental course and clinical outcome in the total sample

\begin{tabular}{|c|c|c|c|c|}
\hline & \multicolumn{2}{|c|}{ Positive symptoms } & \multirow{2}{*}{$\begin{array}{l}\text { Negative symptoms } \\
\mathrm{B}^{\mathrm{a}}\end{array}$} & \multirow[b]{2}{*}{$95 \% \mathrm{CI}$} \\
\hline & $\mathrm{B}^{\mathrm{a}}$ & $95 \% \mathrm{CI}$ & & \\
\hline \multicolumn{5}{|c|}{ Transition to psychosis } \\
\hline $\begin{array}{l}\text { Persisting vs } \\
\text { non-persisting }\end{array}$ & $3.32^{*}$ & $0.76 ; 5.89$ & 1.80 & $-0.67 ; 4.27$ \\
\hline \multicolumn{5}{|l|}{ Total distress ${ }^{\mathrm{b}}$} \\
\hline Decreasing & -0.10 & $-0.21 ; 0.00$ & 0.03 & $-0.08 ; 0.14$ \\
\hline Increasing & $0.20^{* *}$ & $0.07 ; 0.33$ & $0.50^{* * *}$ & $0.39 ; 0.60$ \\
\hline Persisting & -0.05 & $-0.20 ; 0.09$ & $0.56^{* * *}$ & $0.42 ; 0.69$ \\
\hline
\end{tabular}


Table 5

Associations between developmental course and functional outcome.

\begin{tabular}{|c|c|c|c|c|}
\hline & \multicolumn{2}{|c|}{ Social functioning } & \multicolumn{2}{|c|}{ Quality of life } \\
\hline & $\mathrm{B}^{\mathrm{a}}$ & $95 \% \mathrm{CI}$ & $\mathrm{B}^{\mathrm{a}}$ & $95 \% \mathrm{CI}$ \\
\hline \multicolumn{5}{|c|}{ Positive symptoms ${ }^{\mathrm{b}}$} \\
\hline Decreasing & -0.93 & $-2.42 ; 0.57$ & -0.46 & $-0.99 ; 0.07$ \\
\hline Increasing & $-4.32^{* * *}$ & $-6.16 ;-2.49$ & $-1.38^{* * *}$ & $-2.04 ;-0.73$ \\
\hline Persisting & $-2.69 * *$ & $-4.71 ;-0.67$ & $-1.20^{* *}$ & $-1.92 ;-0.47$ \\
\hline \multicolumn{5}{|c|}{ Negative symptoms ${ }^{\mathrm{b}}$} \\
\hline Decreasing & $-3.28^{* * *}$ & $-4.73 ;-1.83$ & $-1.11^{* * *}$ & $-1.63 ;-0.59$ \\
\hline Increasing & $-5.43^{* * *}$ & $-6.73 ;-3.88$ & $-2.05^{* * *}$ & $-2.60 ;-1.50$ \\
\hline Persisting & $-9.29^{* * * *}$ & $-11.09 ;-7.49$ & $-3.59^{* * *}$ & $-4.24 ;-2.95$ \\
\hline
\end{tabular}

** $\mathrm{P}<0.01,{ }^{* * *} \mathrm{P}<0.001$

a Corrected for age, sex, clustering at the family-level and the alternate symptom cluster b Stable-low is used as the reference category.

and Barrantes-Vidal, 2015). A third, -not mutually exclusiveexplanation may lie in the relatively high correlation between the positive and negative clusters ( $\mathrm{r}=0.50$ at baseline and 0.54 at follow-up) combined with the relatively low number of individuals with persistent positive symptoms in the current sample (see Table 2a and 2b). When corrected for the course of negative symptoms, part of the variation in positive symptoms is also 'filtered out'. This may have influenced the results for positive symptoms more than for negative symptoms, as there were fewer individuals with persistent positive symptoms. Finally, it is possible that the course of negative symptoms may partly reflect depressive symptoms. Although adequate discriminative validity for (subclinical) depressive and negative symptoms has been demonstrated, they are also found to be highly correlated $(r=0.72)$ (Stefanis et al., 2002). Similarly, in the current study, we found high correlations between (subclinical) negative and depressive symptoms ( $\mathrm{r}=0.71$ at baseline and 0.78 at follow-up).

\subsection{Genetic risk status predicts impairment}

Regarding the association between genetic risk status and impairment, we found sibling status to predict lower levels of social functioning and lower subjective quality of life at follow-up, corrected for age en gender. As far as we are aware, this is the first study that links genetic risk status for psychosis with both clinician-rated (social functioning) and subjective functional impairment (quality of life). Unfortunately, no data on functional outcome were available at baseline for siblings and controls in the GROUP study, so correcting for baseline social functioning and quality of life was not possible. A similar study in the patient sample of the GROUP study, however, showed that correcting for baseline functioning did not change the results (Janssens et al., 2016).

Additionally, we found that sibling status predicts higher overall symptom-related distress at follow-up, when correcting for baseline symptom-related distress, age en gender. This finding is in line with earlier studies associating genetic risk status for psychosis with higher stress-reactivity, e.g. a study by Myin-Germeys et al. (2001), who found intermediate levels of emotional reactivity to stress in firstdegree relatives of patients with psychotic disorders compared to patients and controls, and Lataster et al. (2009b), who found associations between genetic risk status for psychosis, psychotic experiences and reactivity to stress.

In the current study, siblings also showed a trend towards an elevated risk of developing a clinical psychosis, but this effect did not reach statistical significance. This may be attributed to a power problem, due to the relatively low number of individuals who suffered first onset of clinical psychosis in the current sample $(\mathrm{n}=10,8$ siblings and 2 controls). As the expression of psychotic symptoms is known to peak in adolescence and early adulthood and decreases over time (Verdoux et al., 1998), our relatively low rate of transition may have been affected by the rather high mean age of the participants in the current study (see Table 1).

\subsection{Course of psychotic experiences predicts impairment}

In line with our hypothesis, we found increasing and persistent course of both positive and negative symptoms to be associated with lower social functioning and quality of life, when correcting for age, gender and the alternate symptom cluster. This finding is in line with earlier studies regarding symptom course and functional impairment (Cohen and Davis, 2009; Dominguez et al., 2011; Kaymaz et al., 2012; Kwapil et al., 2013; De Loore et al., 2011; Rossler et al., 2007; Wigman et al., 2011b). Additionally, we found that decreasing negative symptoms were associated with more functional impairment at follow-up, compared to individuals with stable low negative symptoms. This suggests that having experienced negative symptoms (in spite of potential 'recovery' from these negative symptoms) may still serve as a risk factor for functional impairment. This is in line with the hypothesis by Dominguez et al. (2010) that negative symptoms may be associated with earlier developmental impairment.

Increasing course of positive and negative symptoms and persisting course of negative symptoms, but not positive symptoms, were found to be associated with overall symptom-related distress. The latter finding may indicate habituation to psychotic experiences in terms of emotional response, but could also be explained by a power problem due to the relatively low number of individuals with persistent positive symptoms in the current sample, as has been mentioned before. Furthermore, we found that persisting positive, but not persisting negative symptoms, were independently associated with transition to clinical psychosis. This may be explained by construct overlap between subclinical and clinical psychosis, and the well documented fact that clinical psychosis is usually preceded by persistent subclinical positive symptoms (Cougnard et al., 2007; Dominguez et al., 2011; Wigman et al., 2011a; Wigman et al., 2011b).

4.4. No interaction between genetic risk status and course for levels of impairment

In contrast with our expectation, no significant interaction effect was found between genetic risk status and course in the prediction of impairment, indicating that once an individual experiences persistent symptoms, both siblings and controls are equally at risk of developing clinical (in terms of symptom-related distress) and functional impairment. This finding underscores the validity of the pronenesspersistence-impairment model, in individuals with both higher and lower genetic risk for psychosis.

\subsection{Methodological considerations}

Several potential limitations of the current study have been touched on earlier in the Discussion section. For one, some of the findings of the current study may have been limited by statistical power problems, due to the relatively low number of individuals who experienced persistent positive symptoms and who transitioned to clinical psychosis in the current sample. Other aforementioned potential limitations are the lack of correction for (the course of) depressive symptoms mainly in regard to (the course of) negative symptoms, and the lack of feasibility to correct for baseline functional impairment.

Furthermore, phenomenology alone using a self-report measure may not fully capture the vulnerability for schizophrenia-spectrum pathology, as has been mentioned, for instance, by Thaker et al. (1996). Also, developmental course of psychotic experiences was currently assessed at two time-points. Variation in severity of psychotic experiences within-subjects in between time-points is possible. Still, when at two 'random' time-points the severity of psychotic experiences is high, it is likely that these experiences are more persistent over time (i.e. of high severity all the time or a large percentage of time). Finally, as has been mentioned in the Introduction section, so far many terms have been developed to reflect vulnerability for schizophrenia-spectrum 
pathology, yet general consensus on the operationalization of terms is lacking. In response to the this issue, authors have recently called for the need of development of a multidimensional model unifying all symptoms constructs that represent vulnerability for schizophrenia-spectrum pathology (Kwapil and Barrantes-Vidal, 2015). The results of the current study underline the importance of assessing psychotic experiences in the context of genetic risk, multidimensional (including both subclinical positive and negative symptoms) and over time.

\section{Role of funding source}

The GROUP-project is supported by a grant from the Geestkracht program of the Dutch Health Research Council (ZonMw, grant no. 10-000-1002). ZonMw had no further role in the study design, in the collection, analysis and interpretation of data, in the writing of the present paper and in the decision to submit the paper for publication.

\section{Conflict of interest}

None.

\section{Acknowledgement}

We are grateful for the generosity of time and effort by the GROUP participants, and al researchers from participating universities and mental health care organizations who make this GROUP project possible (Amsterdam: Academic Medical Center and the mental health institutions: Ingeest, Arkin, Dijk en Duin, Rivierduinen, Erasmus Medical Center, GGZ Noord Holland Noord. Maastricht: Maastricht University Medical Center and the mental health institutions: GGZ Eindhoven, GGZ Midden-Brabant, GGZ Oost-Brabant GGZ Noord-Midden Limburg. Mondriaan Zorggroep, Prins Clauscentrum Sittard, RIAGG Roermond, Universitair Centrum Sint-Jozef Kortenberg, CAPRI University of Antwerp, PC Ziekere Sint-Truiden, PZ Sancta Maria Sint-Truiden, GGZ Overpelt, OPZ Rekem. Groningen University Medical Center Groningen and the mental health institutions: Lentis, GGZ Friesland, GGZ Drenthe, Dimence, Mediant, GGZ De Grote Rivieren and Parnassia psychomedical center (The Hague). Utrecht: University Medical Center Utrecht and the mental health institutions Altrecht, Symfora, Meerkanten, Riagg Amersfoort en Delta).

\section{References}

Andreasen, N.C., Flaum, M., Arndt, S., 1992. The Comprehensive Assessment of Symptoms and History (CASH): an instrument for assessing diagnosis and psychopathology. Arch. Gen. Psychiatry 49 (8), 615-623.

APA, 2000. Diagnostic and Statistical Manual of Mental Disorders, Fourth Edition, Tex Revision Ed. Washington, DC.

Birchwood, M., Smith, J., Cochrane, R., Wetton, S., Copesake, S., 1990. The social functioning scale. The development and validation of a new scale of social adjustment for use in family intervention programmes with schizophrenic patients. Br. J. Psychiatry 157, 853-859.

Cohen, S., Davis, T.E., 2009. Quality of life across the schizotypy spectrum: findings from a large nonclinical sample. Compr. Psych. 50, 408-414.

Cougnard, A., Marcelis, M., Myin-Germeys, I., De Graaf, R., Vollebergh, W., Krabbendam, L. Lieb, R., Wittchen, H.U., Henquet, C., Spauwen, J., Van Os, J., 2007. Does normal developmental expression of psychosis combine with environmental risk to cause persistence of psychosis? A psychosis proneness-persistence model. Psychol. Med. 37 (4), 513-527.

De Loore, E., Gunther, N., Drukker, M., Feron, F., Sabbe, B., Deboutte, D., van Os, J., Myin-Germeys, I., 2011. Persistence and outcome of auditory hallucinations in adolescence: a longitudinal general population study of 1800 individuals. Schizophr. Res. 127 (1-3), 252-256.

Demjaha, A. Valmaggia, L., Stahl, D., Byrne, M., McGuire, P., 2012. Disorganization/cognitive and negative symptom dimensions in the at-risk mental state predict subsequent transition to psychosis. Schizophr. Bull. 38 (2), 351-359.

Dominguez, M.D., Saka, M.C., Lieb, R., Wittchen, H.U., van Os, J., 2010. Early expression of negative/disorganized symptoms predicting psychotic experiences and subsequent clinical psychosis: a 10-year study. Am. J. Psychiatry 167 (9), 1075-1082.

Dominguez, M.D.G., Wichers, M., Lieb, R., Wittchen, H.U., van Os, J., 2011. Evidence that onset of clinical psychosis is an outcome of progressively more persistent subclinical psychotic experiences: an 8-year cohort study. Schizophr. Bull. 37 (1), 84-93.

Fanous, A., Gardner, C., Walsf, D., Kendler, K.S., 2001. Relationship between positive and negative symptoms of schziophrenia and schizotypal symptoms in nonpsychotic relatives. Arch. Gen. Psychiatry 58 (7), 669-673.

Hanssen, M., Bak, M., Bijl, R., Vollebergh, W., van Os, J., 2005. The incidence and outcome of subclinical psychotic experiences in the general population. Br. J. Clin. Psychol. 44 (2), 181-191.

Janssens, M., Heering, H.D., Boyette, L.-L., van Haren, N.E.M., G.R.O.U.P. investigators, 2016 Persistence of Symptoms Associated With Functional Outcome in Psychosis: A Longitudinal Study, Unpublished Results.

Kaymaz, N., Drukker, M., Lieb, R., Wittchen, H.U., Werbeloff, N., Weiser, M., Lataster, T., van Os, J., 2012. Do subthreshold psychotic experiences predict clinical outcomes in unselected non-help-seeking population-based samples? A systematic review and metaanalysis, enriched with new results. Psychol. Med. 42 (11), 2239-2253.
Kendler, K.S., McGuire, M., Gruenberg, A.M., Walsh, D., 1995. Schizotypal symptoms and signs in the Roscommon family study. Their factor structure and familial relationship with psychotic and affective disorders. Arch. Gen. Psychiatry 52 (4), 296-303.

Konings, M., Bak, M., Hanssen, M., van Os, J., Krabbendam, L., 2006. Validity and reliability of the CAPE: a self-report instrument for the measurement of psychotic experiences in the general population. Acta Psychiatr. Scand. 114 (1), 55-61.

Korver, N., Quee, P.J., Boos, H.B., Simons, C.J., de Haan, L., 2012. Genetic Risk and Outcome of Psychosis (GROUP), a multi-site longitudinal cohort study focused on geneenvironment interaction: objectives, sample characteristics, recruitment and assessment methods. Int. J. Methods Psychiatr. Res. 21 (3), 205-221.

Krabbendam, L., van Os, J., 2005. Schizophrenia and urbanicity: a major environmental influence-conditional on genetic risk. Schizophr. Bull. 31 (4), 795-799.

Kwapil, T.R., Barrantes-Vidal, N., 2015. Schizotypy: looking back and moving forward. Schizophr. Bull. 41 (2), 366-373.

Kwapil, T.R. Gross, G.M. Silvia, PJ. Barrantes-Vidal, N. 2013. Prediction of psychopathology and functional impairment by positive and negative schizotypy in the Chapmans' ten-year longitudinal study. J. Abnorm. Psychol. 122 (3), 807-815.

Lataster, T., Myin-Germeys, I., Derom, C., Thiery, E., van Os, J., 2009a. Evidence that selfreported psychotic experiences represent the transitory developmental expression of genetic liability to psychosis in the general population. Am. J. Med. Genet. B Neuropsychiatr. Genet. 150B (8), 1078-1084.

Lataster, T., Wichers, M., Jacobs, N., Mengelers, R., Derom, C., Thiery, E., Van Os, J., MyinGermeys, I., 2009b. Does reactivity to stress cosegregate with subclinical psychosis? A general population twin study. Acta Psychiatr. Scand. 119 (1), 45-53.

Lataster, T., Verweij, K., Viechtbauer, W., GROUP, 2014. Effect of illness expression and liability on familial associations of clinical and subclinical psychosis phenotypes. Acta Psychiatr. Scand. 129, 44-53.

Linscott, R.J., van Os, J., 2013. An updated and conservative systematic review and metaanalysis of epidemiological evidence on psychotic experiences in children and adults: on the pathway from proneness to persistence to dimensional expression across mental disorders. Psychol. Med. 43 (6), 1133-1149.

Maric, N., Myin-Germeys, I., Delespaul, P., de Graaf, R., Vollebergh, W., Van Os, J., 2004. Is our concept of schizophrenia influenced by Berkson's bias? Soc. Psychiatry Psychiatr. Epidemiol. 39 (8), 600-605.

Moore, T.H., Zammit, S., Lingford-Hughes, A., Barnes, T.R., Jones, P.B., Burke, M., Lewis, G., 2007. Cannabis use and risk of psychotic or affective mental health outcomes: a systematic review. Lancet 370 (9584), 319-328.

Myin-Germeys, I., van Os, J., Schwartz, J.E., Stone, A.A., Delespaul, P.A., 2001. Emotional reactivity to daily life stress in psychosis. Arch. Gen. Psychiatry 58, 1137-1144.

Read, J., Van Os, J., Morrison, A.P., Ross, C.A., 2005. Childhood trauma, psychosis and schizophrenia: a literature review with theoretical and clinical implications. Acta Psychiatr. Scand. 112, 330-350.

Rossler, W., Riecher-Rossler, A., Angst, J., Murray, R., Gamma, A., Eich, D., van Os, J., Gross, V.A., 2007. Psychotic experiences in the general population: a twenty-year prospective community study. Schizophr. Res. 92 (1-3), 1-14.

Statacorp, 2011. Stata Statistical Software: Release 12. Statacorp LP, College Station, TX.

Stefanis, N.C., Hanssen, M., Smirnis, N.K., Avramopoulos, D.A., Evdokimidis, I.K., Stefanis, C.N., Verdoux, H., Van Os, J., 2002. Evidence that three dimensions of psychosis have a distribution in the general population. Psychol. Med. 32 (2), 347-358.

Thaker, G.K., Cassady, S., Adami, H., Moran, M., Ross, D.E., 1996. Eye movements in spectrum personality disorders: comparison of community subjects and relatives of schizophrenic patients. Am. J. Psychiatry 153 (3), 362-368.

Valmaggia, L.R., Stahl, D. Yung A.R. Nelson, B. Fusar-Poli, P., McGorry, P.D., McGuire, P.K. 2013. Negative psychotic symptoms and impaired role functioning predict transition outcomes in the at-risk mental state: a latent class cluster analysis study. Psychol. Med. 43 (11), 2311-2325.

Van Nierop, M., Janssens, M., Bruggeman, R., Cahn, W., de Haan, L., Kahn, R.S., Meijer, C.J. Myin-Germeys, I., van Os, J., Wiersma, D., 2013. Evidence that transition from health to psychotic disorder can be traced to semi-ubiquitous environmental effects operating against background genetic risk. PLoS One 8 (11).

Van Os, J., Linscott, R.J., Myin-Germeys, I., Delespaul, P., Krabbendam, L., 2009. A systematic review and meta-analysis of the psychosis continuum: evidence for a psychosis proneness-persistence-impairment model of psychotic disorder. Psychol. Med. 39 (2), 179-195.

Verdoux, H., van Os, J., Maurice-Tison, S., Gay, B., Salamon, R., Bourgeois, M., 1998. Is early adulthood a critical developmental stage for psychosis proneness? A survey of delusional ideation in normal subjects. Schizophr. Res. 29 (3), 247-254.

WHO, 1998. Development of the World Health Organization WHOQOL-BREF quality of life assessment. The WHOQOL group. Psychol. Med. 28 (3), 551-558.

Wigman, J.T., van Winkel, R., Jacobs, N., Wichers, M., Derom, C., Thiery, E., Vollebergh, W.A., van Os, J., 2011a. A twin study of genetic and environmental determinants of abnormal persistence of psychotic experiences in young adulthood. Am. J. Med. Genet. B Neuropsychiatr. Genet. 156B (5), 546-552.

Wigman, J.T., van Winkel, R., Raaijmakers, Q.A., Ormel, J., Verhulst, F.C., Reijneveld, S.A., van Os, J., Vollebergh, W.A., 2011b. Evidence for a persistent, environmentdependent and deteriorating subtype of subclinical psychotic experiences: a 6-year longitudinal general population study. Psychol. Med. 41 (11), 2317-2329. 